\title{
A chronic multi-electrode microdrive for small animals
}

\author{
Jeff G. Keating *, George L. Gerstein \\ Department of Neuroscience, University of Pennsylvania, A304 Richards Building, 3700 Hamilton Walk, Philadelphia, PA 19104-6085, USA
}

Received 7 December 2001; received in revised form 22 April 2002; accepted 22 April 2002

\begin{abstract}
We describe a simple microdrive device appropriate for chronic microelectrode recording in rats. No precision machining is required; all parts are stock or cut from standard stock material with hand tools and assembled with epoxy. The device together with its electrodes can be discarded at the completion of the experiment. (C) 2002 Published by Elsevier Science B.V.
\end{abstract}

Keywords: Microdrive device; Microelectrode; Standard stock material

\section{Introduction}

Three different basic drive designs have been used in microdrives for use in recording from small animals: hydraulic based (Veregge and Frost, 1985), gear based (Sinnamon and Woodword, 1977), and screw based; one of the earliest of the latter is that of Ranck (1973). His microdrive consisted of two screws, one mounted inside the hollowed out other. The outer screw was used to attach the microdrive to nuts mounted on the animal's head, and the inner screw held the electrode. When the inner screw was turned, the electrode would advance, albeit turning itself. The design was simple and economical. However, the difficulty of centering the electrode, and damage resulting from its twisting on its way through the brain, led others to improve upon its design. A principal variant involved adding another concentric screw and a key, constructed in such a way as to advance the electrode without turning it (Goldberg et al., 1993; Deadwyler et al., 1979; Bland et al., 1990). A second variant involved holding the electrode off axis and attaching it in such a way that it moved up and down with the turning of the drive screw (Ainsworth and O'Keefe, 1977; Felsten et al., 1987; Harper and McGinty, 1973; Zhang and Harper, 1984).

At some point in the construction of all the simple designs described above, some machining and/or highly

\footnotetext{
* Corresponding author. Tel.: +1-215-898-8752; fax: +1-215-5735851

E-mail address: jeff@mulab.physiol.upenn.edu (J.G. Keating).
}

accurate drilling is required. Some of the best, more complex, designs require extensive machining (e.g. Pager, 1984; Venkatachalam et al., 1999). In contrast, the design described here involves no machining, only cutting and drilling one hole, all of which can be performed by the researcher using hand held tools. The critical dimensions of the drive, including the capture and alignment of the drive screw, have been obtained by the selection of stock material and the method of construction.

\section{Materials and assembly}

The raw materials and sources required for construction of the microdrive are listed in Table 1. It is essential to obtain the precise set of stock dimensions; however it may be possible to find other part size combinations that would also work. In addition to the parts listed in Table 1, superglue and epoxy are used to hold the parts together, and silver print paint and solder are used to fix the electrodes to the connector with a good electrical connection. The microdrive has three subassemblies: the drive unit, the alignment unit, and the case. Electrodes are threaded through the alignment unit, and soldered to a connector. The alignment unit is then attached to the drive unit. Finally, the entire mechanism is enclosed in the case. We describe a design that allows $2 \mathrm{~mm}$ of movement for the electrodes, sufficient for our recording needs in rat neocortex. The design can be easily extended in length if greater recording depth is required. 
Table 1

Parts

\begin{tabular}{ll}
\hline Number & Item \\
\hline 1 & $\begin{array}{l}0 / 80 \times 3 / 8 \text { in. slotted flat head machine screw } 18-8 \text { stainless } \\
\text { steel }\end{array}$ \\
2 & $0 / 80$ machine screw nut $18-8$ stainless steel \\
2 & Size \#0 flat nylon washers \\
2 & M1.6 washers $18-8$ stainless steel (Metric: ID $0.5 \mathrm{~mm}$ OD 1.6 \\
& mm) \\
1 & Size 003 buna o-ring \\
1 & $3 / 32 \times 3 / 16$ in. brass rectangular tubing \\
1 & $1 / 8 \times 1 / 4$ in. brass rectangular tubing \\
1 & $5 / 32 \times 5 / 16$ in. brass rectangular tubing \\
1 & $3 / 16 \times 3 / 8$ in. brass rectangular tubing \\
1 & $0.220 \times 0.340$ in. brass rectangular tubing \\
2 & 12 in. pieces of 31 gauge stainless steel tubing \\
8 & $4 \mathrm{~cm}$ long 50 um formvar insulated stainless steel wires \\
1 & GF-10 connector \\
\hline
\end{tabular}

The parts are standard sizes and readily available from a variety of sources. We purchased the screws (part number 91792 A052), nuts (part number 91841 A115), and buna o-rings (9452 K11) from McMaster-Carr, New Brunswick, NJ, metric washers (part number c6rhflg) from Allmetal screw products corporation, brass rectangular tubing (assortment \#Q-RCTB-100), nylon washers (part number WN0 ) and stainless steel tubing (part number HTX-31-24) from Small Parts, Miami Lakes, FL, the GF-10 connector from Microtech, Inc., Boothwyn, PA. The wire (50 um stainless steel 304, H-formvar insulated) was purchased from California Fine Wire, Grover Beach, CA, alternatively sharp metal electrodes (part number UESSCESECTNJ) were purchased from FHC, Inc. (Frederick Haer and Company) Bowdoinham, ME.

\subsection{Drive unit instructions}

The drive unit is constructed of a screw, two nuts, two steel and two nylon washers, an o-ring and a piece of cut rectangular tubing (Fig. 1A). The electrodes will be attached to one of the nuts, which is captive in the cut piece of rectangular tubing, and moves along the shaft of the screw as the screw is turned. To start construction, cut the $3 / 16 \times 3 / 8$ in. brass rectangular tubing down the middle of the $3 / 8 \mathrm{in}$. faces so as to make two $3 / 16$ in. wide ' $c$ ' channels. Cut a $4.0 \mathrm{~mm}$ long piece from a ' $c$ ' channel and debur it ( $4.0 \mathrm{~mm}$ maximizes the drive distance for the specified screw length). Place a nylon washer, and then a steel washer, on the screw. Thread a nut to about two thirds of the way to the head of the screw. Rotate the hexagonal nut as needed so that two of its sides align with the sides of the brass channel. Place this assembly in the brass channel so that the nut fits snugly inside, forming a perfect friction fit. The steel washer should lie flat with its edges resting on the edges of the ' $c$ ' channel. Place the second steel washer on the screw so that it rests against the other end of the brass ' $c$ ' channel. Place the o-ring, and the second nylon washer on the screw. Finally, thread on the second nut and tighten so that the o-ring is snug, but not overly compressed. Align the steel washers, so that they lie symmetrically on each end of the channel (they should just overlap all three edges of the brass channel on both ends). Wet the bottom nut and screw with super glue, also touch the four corners where the steel washers rest on the brass channels with super glue.

The brass channel supports the washers holding the screw, at the same time channeling the nut so that it does not rotate, nor wobble. The inner diameter of the steel washers is just slightly larger than the diameter of the screw, so the screw rotates freely. The bottom nut is outside the channel, and rotates freely together with the screw. Tightening the nut slightly during construction compresses the o-ring, pulling the conical neck of the flat head screw tightly against the inside of the steel washer, holding the entire assembly together, and minimizing what little center wobble the steel washer alone would allow. The nylon washers minimize friction, allowing the screw to turn smoothly with minimal force. The drive unit slides snugly inside the next larger piece of telescoping brass tubing, which will be used as the case.

\subsection{Alignment unit instructions}

The alignment unit is constructed of eight pieces of stainless steel tubing and two brass ' $c$ ' channels which, when placed on either side of the stainless steel tubes and forced together, align the stainless steel tubes into a tight, linear, array (Fig. 1B). The electrodes will later be threaded through the tubes, aligning them with a center to center electrode spacing of $250-300 \mathrm{um}$. To start construction, cut the $1 / 8 \times 1 / 4 \mathrm{in}$. and the $3 / 32 \times 3 / 16$ in. brass rectangular tubing down the middle of their wider side (i.e. cut along the $1 / 4$ and $3 / 16$ in. faces) making long ' $c$ ' channels. Cut pieces $5 \mathrm{~cm}$ in length from of those telescoping ' $c$ ' channels. Cut the 31 gauge stainless steel tubing into $7 \mathrm{~cm}$ lengths and lay eight lengths flat, parallel, and tight up against each other. Hold with masking tape across the last $5 \mathrm{~mm}$ or so of each end of the bundle. Put a layer of freshly mixed epoxy inside the wider ' $c$ ' channel. Lay the steel tubes flat inside, ensuring epoxy covers the entire surface. Next insert the narrower ' $c$ ' channel so that the base presses the stainless steel tubing flat. Squeeze the brass 'c' channels together tightly, pressuring the tubing parallel to the channel. Epoxy should come out the ends. Clamp until the epoxy is set.

Cut a $5 \mathrm{~mm}$ piece of the glued brass 'c' channelstainless steel tubing assembly. Debur the exposed ends of the stainless steel tubes and make sure they are clear by reaming them. During deburring, the tubes may come loose if the alignment unit is cut shorter than 5 $\mathrm{mm}$. Place an alignment unit in a Pyrex dish filled with white petroleum jelly; heat the dish over a low flame until the jelly melts and the unit falls to the bottom. Continue heating until no further bubbles come out of 
A. Drive unit construction

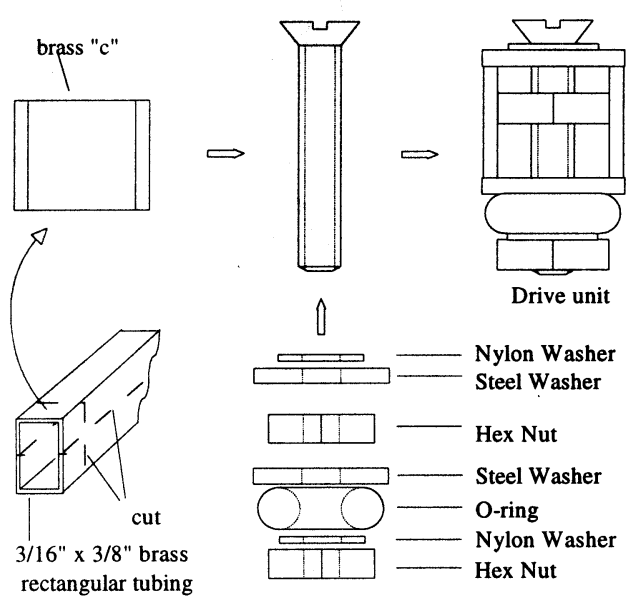

C. Case construction
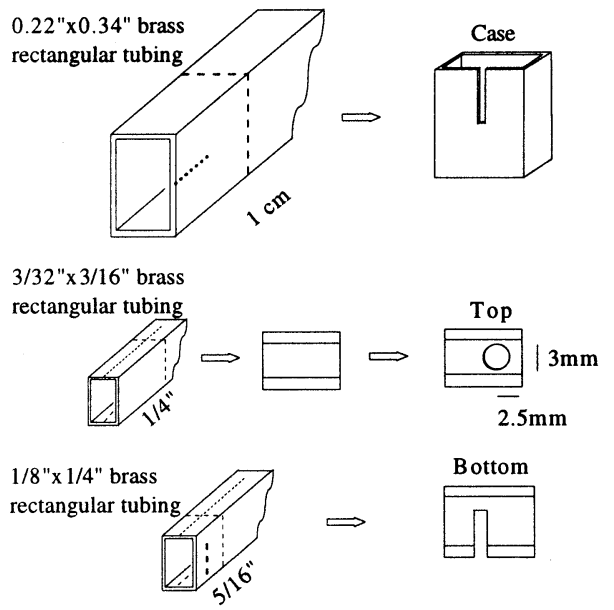

\section{B. Alignment unit construction}

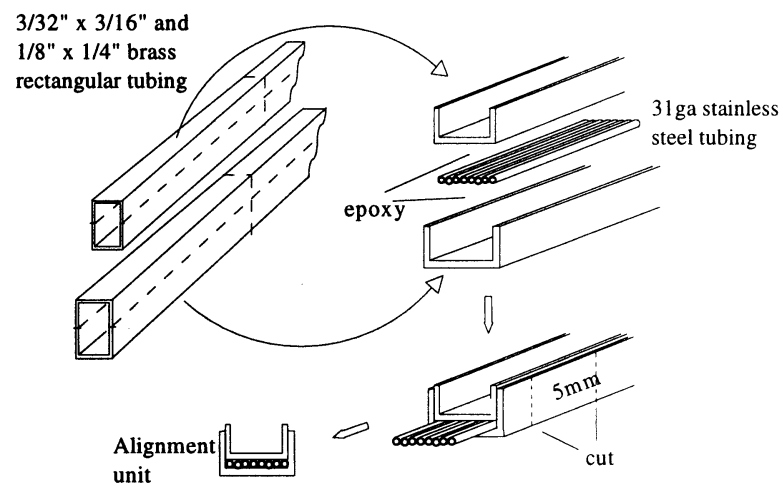

D. Assembly
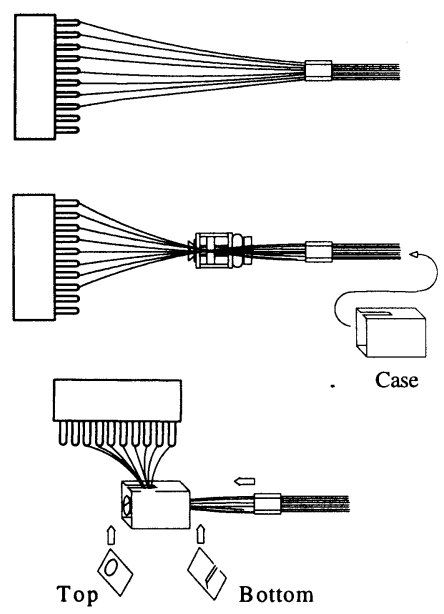

Fig. 1. (A) The drive unit construction process. (B) The alignment unit construction process. (C) The case construction process. (D) The assembly process. Dotted lines indicate where cuts are made. See text for details.

the tubing. Let cool until the jelly solidifies. Remove the alignment unit and wipe clean. The petroleum jelly is needed to seal the tubes and keep CSF from seeping in post-implantation, crystallizing, and freezing the electrodes in position.

The microdrive design permits attachment of other alignment units; minor modifications in the above procedure can be used to produce a hexagonal seven tube array rather than the linear array of eight guide tubes and electrodes.

\subsection{Case instructions}

The case is constructed from three pieces cut from brass rectangular tubing (Fig. 1C). The body is a $1 \mathrm{~cm}$ long piece of the $0.220 \times 0.340$ in. brass rectangular tubing. Cut a $1 \mathrm{~mm}$ wide slot half way down one of the wide sides to enable the electrodes to exit the case. For the top of the case cut a $1 / 4 \mathrm{in}$. piece of the $3 / 32 \times 3 / 16$ in. brass rectangular tubing and then cut down the middle of the $3 / 32$ in. sides. This gives two pieces that are $1 / 4$ in. by $3 / 16$ in., with a $3 / 64 \mathrm{in}$. lip on two sides. Drill a $3 \mathrm{~mm}$ diameter hole $2.5 \mathrm{~mm}$ from the end of one of these pieces. The piece will fit into the case body, rest on the nylon washer and clear the head of the drive screw. To make the bottom of the case, cut a 5/16 in. piece of the $1 / 4 \times 1 / 8$ in. rectangular tubing. Remove one of the $1 / 4 \mathrm{in}$. faces by cutting along each of the $1 / 8$ in. sides. The piece should be $5 / 16$ by $1 / 4$ in. with a $1 \mathrm{~mm}$ lip on two sides. The lips will allow the piece to snap into place along the outside of the bottom of the case. Make a $1 \mathrm{~mm}$ wide cut $2 / 16 \mathrm{in}$. in along one of the $5 / 16 \mathrm{in}$. sides and going $3 / 4$ of the way across the piece. The slit enables the electrodes to pass through, exiting the case. 

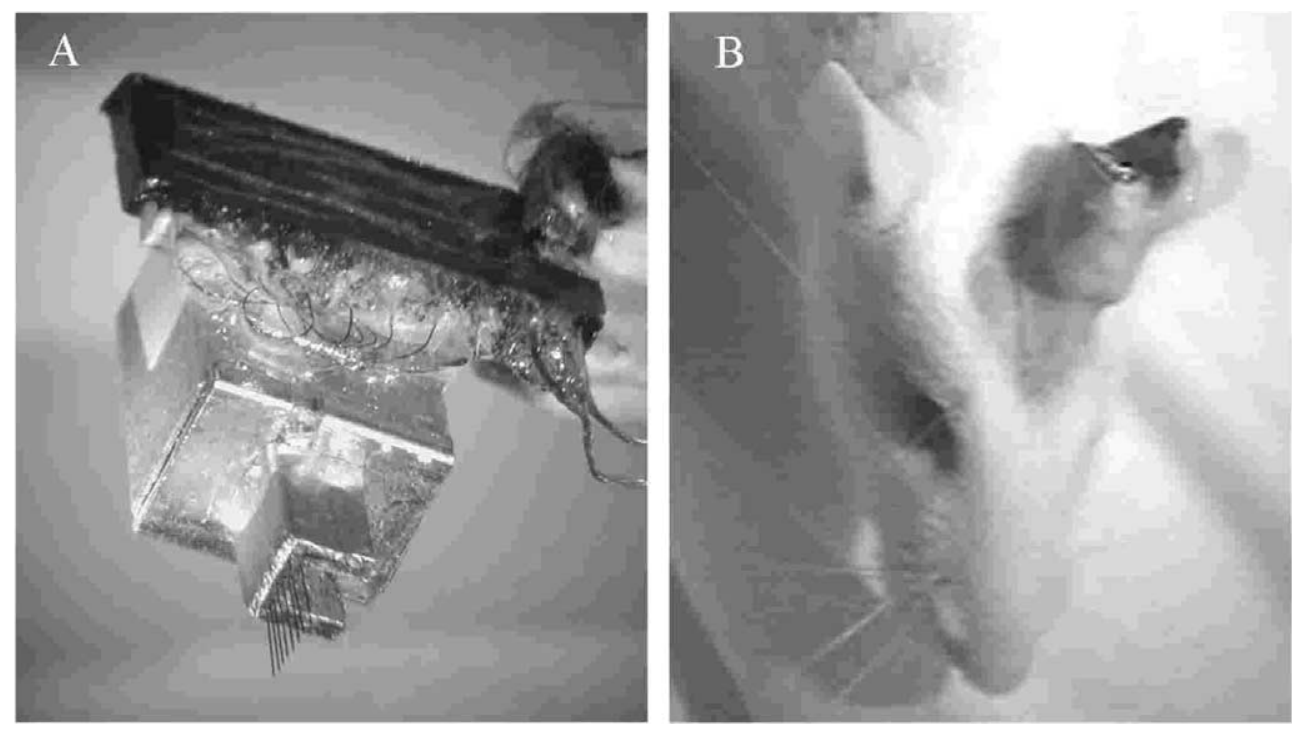

Fig. 2. The assembled microdrive. (A) View of the enclosed drive, with the microwire electrodes exiting the alignment unit. The black plastic connector has been epoxied to the side of the drive case. Larger wires, one used for grounding the animal and the other as a reference for the differential amplifiers, have been added to the connector. (B) The microdrive installed on a rat.

\subsection{Assembling the drive}

To assemble the microdrive, first the electrodes are threaded through the alignment unit and attached to the connector. Next, this electrode-alignment unit piece is attached to the drive unit. Finally, the drive and electrodes are placed in the case and the assembly is sealed (Fig. 1D).

To start assembly, thread the wires or sharp metal electrodes through the alignment unit. Using the appropriate flux, solder each wire or electrode to the connector. Apply silver print paint to each solder joint. On rare occasions, the solder connection alone may fail with time; the silver print paint ensures a good electrical connection. After allowing the silver print paint to dry, encase the connections in epoxy. For tungsten wires, solder will hold the wire in place while the silver print paint dries and ensures electrical contact.

To attach the electrodes to the drive unit, rotate the alignment unit $180^{\circ}$ axially while holding the connector steady. This will cause the electrodes to cross over each other at one point (see Fig. 1D middle). Slide the alignment unit up along the electrodes until this crossing point is about $1.5 \mathrm{~cm}$ from the connector. Place this crossing point over the drive nut of the drive unit and fasten the electrode bundle to the nut with epoxy. Using a needle, spread an additional very thin layer of epoxy along the edges and sides of the nut to ensure a solid grip. If wires are used, the ends are aligned after construction is complete by evenly cutting off the ends with a sharp scissors. If prefabricated electrodes are used, the tips must be carefully aligned at this point, before the glue dries.

Place a small drop of epoxy on the inside of a narrow edge of the $1 \mathrm{~cm}$ long piece of the $0.220 \times 0.340 \mathrm{in}$. brass rectangular tubing that was cut for the case body. Slide the alignment and drive unit through the case body, entering through the side where the slit has been cut. Place the drive unit on the epoxy drop to fix it in the case body. Adjust the position of the drive until the bottom of the screw is just inside the lower edge of the case body. Let the epoxy set. Slide the upper portion of the wires or electrodes through the slit in the side of the case body, and super glue the connector to the side. Make sure to leave a length of loose wire inside the case body so the electrodes can advance as the nut moves along the screw. Place the case top so that the head of the screw pokes out of the hole and let the top rest on the nylon washer. Epoxy the edges in place. Align the slot in the case bottom piece with the electrodes, slide it between the case body and the alignment unit, and snap it onto the case body. Slide the alignment unit up against the case bottom piece and epoxy it, and the bottom piece, into place. Seal up any remaining holes and cover the exposed electrode wires between the case and connector with epoxy. If wires are being used as electrodes, cut the ends off evenly, leaving approximately $0.5 \mathrm{~mm}$ extending from the end of the alignment unit. The assembled microdrive (Fig. 2) weighs $2.2 \mathrm{~g}$. This weight does not appear to encumber our rats; other investigators have claimed that rats can support up to 25 $\mathrm{g}$ (Fee and Leonardo, 2001). 
A

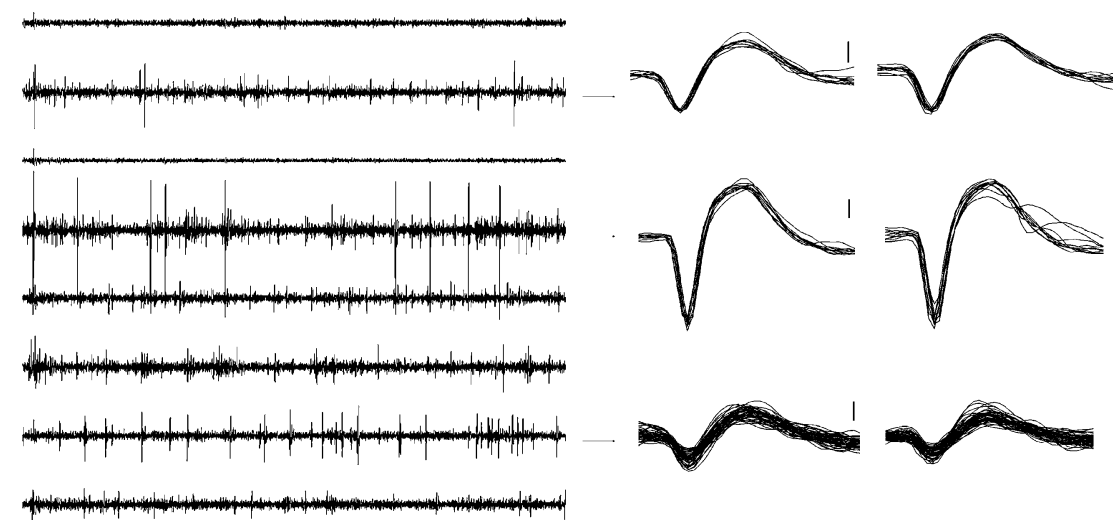

Fig. 3. Electrical activity recorded in the auditory neocortex of an awake behaving rat. (A) Shows a $1 \mathrm{~s}$ period of activity collected using the drive. Data were taken from a $20 \mathrm{~min}$ recording session. Channels are numbered from bottom to top. (B) Shows the first ten sorted spikes on channels 7 and 5 (counted from the bottom up in A) and the first 40 spikes $>200$ uv on channel 2. (C) Shows the last ten sorted spikes on channels 7 and 5 , and the last 40 spikes $>200$ uv on channel 2. Although the waveforms in channel 2 are large, they are not sortable. Scale bars in B are 100 uv; waveforms in $\mathrm{C}$ are shown using the same scale. The drive was built with sharp stainless steel microelectrodes (FHC electrodes \#UESSCESECTNJ), custom built headstage preamplifiers, Plexon amplifiers, and data acquisition with a MultiChannel Systems Board and software.

\section{Installing the microdrive}

The microdrive has been designed for chronic recording. All procedures described have been carried out in accordance with NIH guidelines and approved by the Institutional Animal Care and Use Committee of the University of Pennsylvania.

Once the animal is anesthetized and all tissues over the skull reflected, three holes are drilled through the outer table of the skull with a $0.8 \mathrm{~mm}$ head size round burr for anchor screw placement. Pan head screws (1/8 in. 0/80) are placed in the holes by turning two full rotations. This secures the screw without going so deep as to compress the underlying dura. A ground wire is attached to two of the screws, a reference wire is attached to the third. An area of the skull $0.6 \mathrm{~mm}$ wide by $2.5 \mathrm{~mm}$ long is removed using a surgical drill and a $0.6 \mathrm{~mm}$ head size round burr. To minimize damage to the area to be recorded, both from cutting the overlying dura and from the destruction of the columnar organization of the cortex, the brain is entered at a $45^{\circ}$ angle. The outer table of the skull is removed near the craniotomy to allow the microdrive unit a closer approach; minimizing the length of exposed electrodes. A slit is made in the dura where the electrodes will enter. A sterotaxic device is used to position the microdrive into place. While viewing through a surgical microscope, the entire microdrive assembly is advanced with a stepper motor attached to a stereotaxic apparatus. We start with the electrodes extending from the alignment unit $0.5 \mathrm{~mm}$. The drive is advanced until the alignment unit is just about to touch the skull or dura. This moves the electrodes about $350 \mathrm{um}$ into the cortex. If the hole through which the electrodes are placed in the brain has been enlarged (for example, to avoid a blood vessel) the opening is covered with gelfoam to protect it from acrylic. If the hole remains small, it will fill with $\mathrm{CSF}$, protecting the electrodes and the brain from the acrylic layer, which will cover it. Cover the screws and exposed skull with a very thin, liquid layer of acrylic. This will allow the acrylic to soak into the smaller pores of the skull forming a good bond. Fixing the microdrive in place with more acrylic also covering the skull and screws finishes the installation.

\section{Using the microdrive}

Following recovery from surgery, we examine the signals, and move the electrodes as necessary to obtain large waveforms on as many electrodes as possible. Turning the drive screw counter clockwise advances the electrodes; turning it clockwise retracts the electrodes. Since a $0 / 80$ screw is used, there are 80 turns per inch, giving 317 um per $360^{\circ}$ turn. If the drive unit is assembled with the right tension, it is easy to make 1/ 16 turn $(20 \mathrm{um})$ or even smaller advances while holding the awake animal in your hand. Following recovery from surgery, or other long periods of time when the electrodes are not moved, often the electrodes will fail to advance on turning the screw. They can be freed by simply backing up $1 / 2$ turn, and then advancing.

Example data is shown in Fig. 3. Fig. 3A shows a $1 \mathrm{~s}$ trace from a linear array of eight electrodes using the microdrive. As is shown in $\mathrm{B}$ and $\mathrm{C}$, two of the electrodes showed clearly isolatable waveforms, which were stable over the course of a recording session. More usual is the situation shown in the next to bottom trace of Fig. 3A and the lowest set of waveforms in B and C. 
Although large, these waveforms are not sortable. The variability in amplitude of the waveforms is not due to relative movement of the electrode and the cell, since all electrodes are fixed together, and such variability is not seen on channels 5 and 7. Rather, the variation is probably due to the discharge of several cells nearly equidistant from electrode 2; such a situation is a common occurrence (Gray et al., 1995).

\section{Acknowledgements}

We thank Dr Pawel Musial for the use of his recordings. This work was performed with funding by NIH grants MH46428 and DC01249.

\section{References}

Ainsworth A., O'Keefe J.. A lightweight microdrive for the simultaneous recording of several units in the awake, freely moving rat. $\mathrm{J}$ Physiol 1977;269(1):8P-10P.

Bland B.H., Colom L.V., Mani T.E.. An improved version of a directdrive, nonrotating manual microdrive. Brain Res Bull 1990;25:441-3.

Deadwyler S.A., Biela J., Rose G., West M., Lynch G.. A microdrive for use with glass or metal microelectrodes in recording from freely-moving rats. Electroencephalogr Clin Neurophysiol $1979 ; 47: 752-4$
Fee M.S., Leonardo A.. Miniature motorized microdrive and commutator system for chronic neural recording in small animals. J Neurosci Methods 2001;112:83-94.

Felsten G., Cook J.A., Thompson M.E., Natelson B.H.. Recordings from arterial pressure-sensitive unit in the awake dog. Brain Res Bull 1987;19:75-80.

Goldberg E., Minerbo G., Smock T.. An inexpensive microdrive for chronic single-unit recording. Brain Res Bull 1993;32:321-3.

Gray C.M., Maldonado P.E., Wilson M., McNaughton B.. Tetrodes markedly improve the reliability and yield of multiple single-unit isolation from multi-unit recordings in cat striate cortex. J Neurosci Methods 1995;63(1-2):43-54.

Harper R.M., McGinty D.J.. A technique for recording single neurons from unrestrained animals. In: Phillips M.I., editor. Brain Unit Activity and Behavior. Springfield, IL: Thomas, 1973:82-104.

Pager J.. A removable head-mounted microdrive for unit recording in the free-behaving rat. Physiol Behav 1984;33:843-8.

Ranck J.R., Jr. A moveable microelectrode for recording from single neurons in unrestrained rats. In: Phillips M.I., editor. Brain Unit Activity During Behavior. Springfield, IL: Thomas, 1973:76-9.

Sinnamon H.M., Woodword D.J.. Microdrive and method for single unit recording in the active rat. Physiol Behav 1977;19:451-3.

Venkatachalam S., Fee M.S., Kleinfeld D.. Ultra-miniature headstage with 6-channel drive and vacuum-assisted micro-wire implantation for chronic recording from the neocortex. J Neurosci Methods 1999;90(1):37-46.

Veregge S., Frost J.D.. A simple, inexpensive, hydraulic microdrive for recording neocortical unit activity in the unanesthetized rat. Electroencephalogr Clin Neurophysiol 1985;61(1):94-7.

Zhang J.X., Harper R.M.. A new microdrive for extracellular recording of single neurons using fine wires. Electroencephalogr Clin Neurophysiol 1984;57:392-4. 